\title{
SDN based Operator Assisted Offloading Platform for Multi-Controller 5G Networks
}

\author{
Madhusanka Liyanage $^{1}$, Mahesh Dananjaya ${ }^{2}$, Jude Okwuibe $^{3}$, Mika Ylianttila ${ }^{4}$ \\ ${ }^{1,3,4}$ Centre for Wireless Communications (CWC), University of Oulu, Finland \\ ${ }^{2}$ WSO2, Paraqum Technologies, Sri Lanka \\ Email: $\left[{ }^{1}\right.$ madhusanka.liyanage, ${ }^{3}$ jude.okwuibe, ${ }^{4}$ mika.ylianttila $] @$ oulu.fi, ${ }^{2}$ dananjayamahesh@gmail.com
}

\begin{abstract}
This paper presents an operator-assisted data offloading platform for 5G mobile networks by using Software Defined Networking (SDN). By enabling lateral communication between multiple SDN controllers, operators are able to perform the offloading process without the intervention of the user. Moreover, the offloading decision of proposed platform is based on accurate real time network conditions. The proposed mechanism is implemented on a testbed to verify feasibility and performance.

Index Terms-5G, Data Offloading, Software Defined Networks, OpenFlow, Scalability, Performance.
\end{abstract}

\section{INTRODUCTION}

The exponential surge in mobile data traffic in recent decade has created a huge pressure for service providers to seek alternative and more efficient ways of handling mobile data traffic and decongesting cellular bands. Systematically transferring data traffic from cellular networks to a complementary network such as Wi-Fi comes as a natural alternative for service providers attempting to meet the traffic demands of present day and future heterogeneous networks [1], [2]. However, most legacy data offloading schemes are triggered by the user who has no proper knowledge of the network condition or by mobile applications which have limited access to full network status indicators. Therefore, user initiated offloading mechanism are less optimal in most cases.

Software Defined Networking (SDN) is one of the key enablers of $5 \mathrm{G}$ mobile networks [2]. SDN primarily moves network control functions away from traditional switches to an external software entity called the controller which controls the network. In SDN based 5G networks, it is possible to enable the exchange of real time network parameters between different operators by enabling real-time bilateral communication between mobile SDN controllers.

In this paper, we propose a novel operator-assisted offloading platform for SDN based $5 \mathrm{G}$ networks. By using the proposed platform, the mobile operator is able to initiate the offloading process without the intervention of the user, hence the offloading decision is taken based on accurate real time network conditions. Moreover, the proposed offloading platform is independent of offloading mechanisms. The mobile operator is able to select any compatible offloading mechanisms and run on top of the proposed offloading platform. Several works have proposed the use of SDN for mobile data offloading [3]-[8]. However, these data offloading mechanisms [3]-[6], [8] are designed for single-operator single-controller networks. For the proposed approach, we consider generic scenario where the underlying platform supports not only the single-operator single-controller networks but also multioperator multi-controller networks.

With the proposed approach, different operators can control different $5 \mathrm{G}$ network segments (i.e. mobile network, Wi-Fi network, Li-Fi Network etc) by using different SDN controllers. Hence to enable the seamless offloading between different operator networks, we propose a new lateral communication mechanism between multiple controllers to exchange user credentials and offload information. The proposed platform can be used to perform offloading between any two complementary networks. However, only the Wi-Fi offloading use case is presented in this paper. A proof of concept of the proposed platform is implemented by using Floodlight SDN controller and the RESTful architecture on Mininet emulator environment. We verify the feasibility of proposed platform and investigate the achievable performance improvement by using the platform.

The rest of this paper is organized as follows. Section II presents the proposed operator-assisted offloading platform and components. Section III contains the experiment testbed and test results. Finally, Section IV concludes the paper.

\section{Operator-Assisted OfFloading Platform}

The proposed mechanism is an operator assisted mechanism where the user is not actively participating in the offloading action. The proposed platform can be used to offload the mobile traffic to any kind of non-3GPP access technology such as Wi-Fi, Li-Fi, Satellite or Wi-Max. However, we have considered only Wi-Fi offloading in this work, hence the rest of this paper will focus on Wi-Fi offloading using the proposed mechanism.

Figure 1 shows the architecture of the offloading platform for multi-controller $5 \mathrm{G}$ networks. This platform consists of several components, however, the data offloading core is the main module that contains all relevant classes and functions required to perform the offloading action. It also manages the exchange of information about users between multiple SDN controllers. The proposed data offloading core is implemented as an application plane program which runs on top of each 


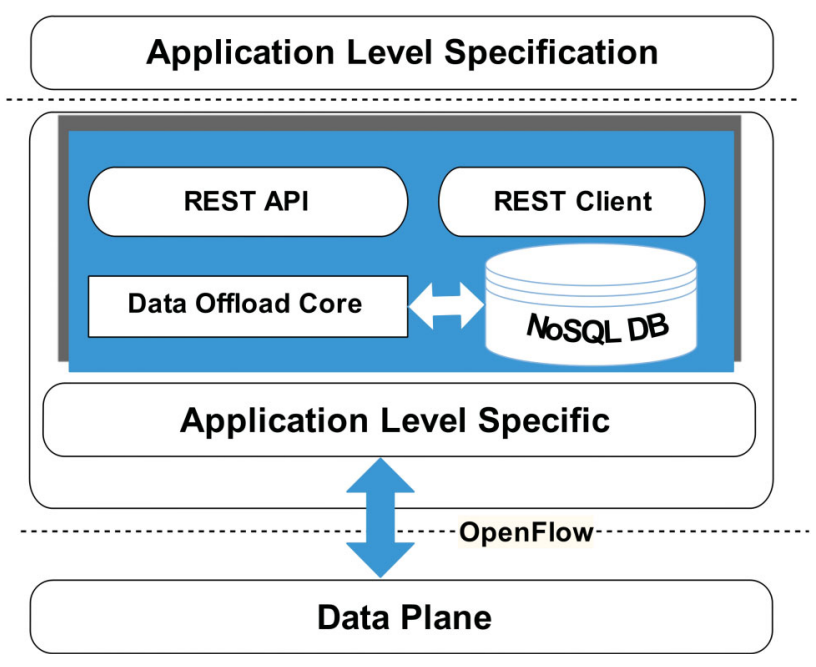

Fig. 1: The Architecture of Offloading Platform

network controller. This core contains separate modules for each offloading scheme. It is possible to add/drop these schemes according to the offloading algorithms used by the operator.

\section{A. Multi-Controller Communication}

In this section, we describe multi-controller communication mechanism of the proposed offloading platform. Figure 2 illustrates the proposed message exchange between different entities to perform the Wi-Fi offloading. The data offloading core (Figure 1) manages the offloading function in each network (i.e. mobile and Wi-Fi networks).

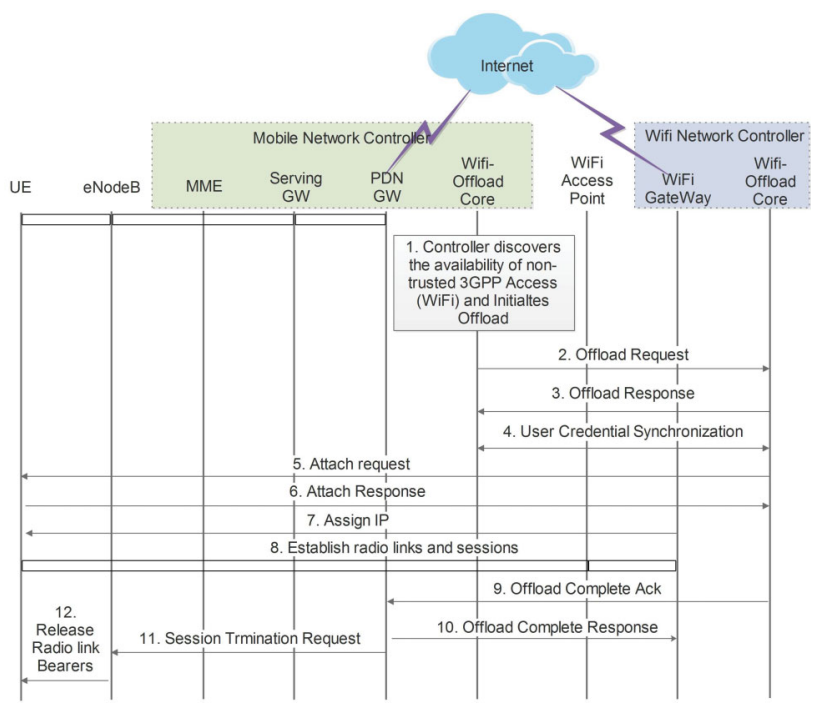

Fig. 2: Multi-Controller Communication

In the first step, the offloading core in mobile network runs pre-installed Wi-Fi offloading mechanism and initiates the offloading process after it identifies the presence of compatible
Wi-Fi networks near to the User Equipment (UE) based on predefined service agreements. In the second step, the offloading core in the mobile network sends an offload request to the offloading core in the Wi-Fi network. If the Wi-Fi network has enough network resources to offer services for UE, the offloading core in the Wi-Fi network sends an offload respond back to the mobile network in the third step. In the fourth step, the offloading cores in the Wi-Fi and mobile networks synchronize the user credential of UE.

Then, offloading core in the Wi-Fi network starts a new connection with the UE by using the already shared user credentials in step 4. It sends an attach request to the UE in step 5 and the UE responds with an attach response in step 6. The IP addresses are assigned in Step 7 and a new session is established in step 8 . Then the offloading core in the Wi-Fi network sends an offload complete Acknowledgment (Ack) to the offloading core in the mobile network (Step 9). The offloading core in the mobile network sends a complete response to the Wi-Fi network (Step 10) and a session termination request to the eNodeB (Step 11). In the last step (Step 12), all the radio link bearers are released by mobile network. Now, the data communication of UE is performed via the Wi-Fi network.

In the scope of this paper, we implement three offloading mechanisms namely; signal strength-based offloading, costbased offloading and subscriber density-based offloading.

\section{TESTBED IMPLEMENTATIONS AND RESULTS}

We implement the proposed offloading platform in a testbed. Figure 3 illustrates the experiment testbed. Here, we used up to 18 users. Each user had an IPERF client which was connected to IPERF server.

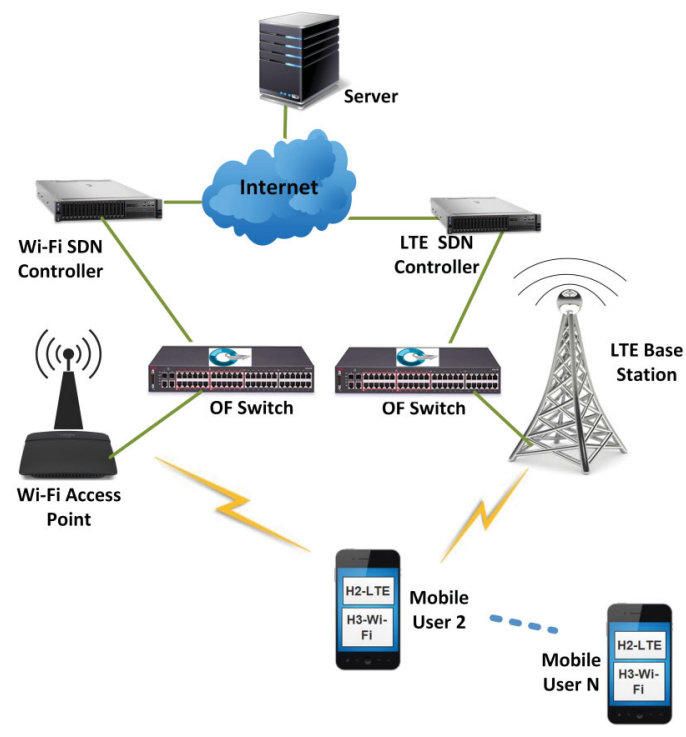

Fig. 3: Experiment Testbed

\section{A. Feasibility of Wi-Fi Offloading Mechanisms}

In this experiment, we verify the interoperability of different offloading mechanisms. Thus, we enabled cost based offload- 
ing mechanism in the mobile controller and subscriber density based offloading mechanism in the Wi-Fi controller. We set the offloading threshold of the Wi-Fi controller to 10 and the cost of $\mathrm{Wi}-\mathrm{Fi}$ is lower than mobile.

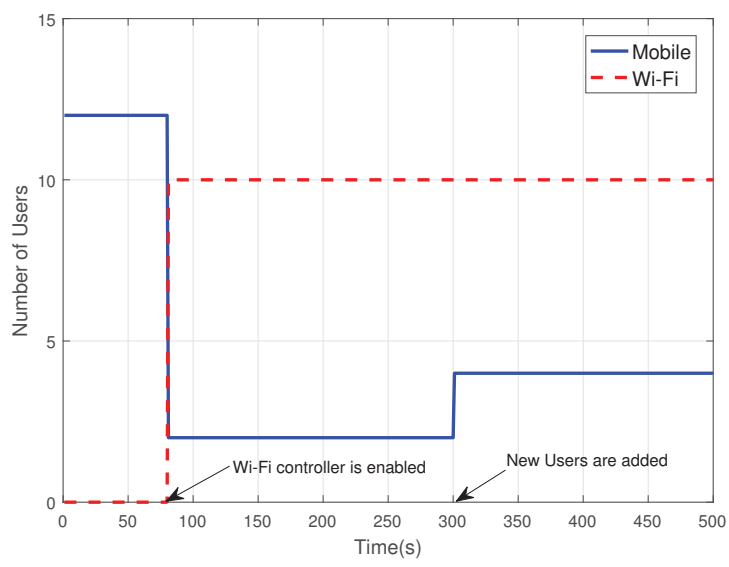

Fig. 4: Number of Users on WiFi and LTE Controllers with the time.

Initially, only the mobile network is available on and all users are connected with the mobile network. Once the WiFi network is functional, most of the users (up to 10) are offloaded to Wi-Fi network. Here, Wi-Fi network did not accept any offload request beyond its threshold (i.e. 10 user). Therefore, the rest of the two user as well as the newly connected users remained in the mobile network. This experiment confirmed that our platform supports the interoperability of different offloading mechanisms.

\section{B. Offload Delay}

In the second experiment, we measured the offloading delay of the proposed platform. We tried 18 offloading attempts (From mobile to $\mathrm{Wi}-\mathrm{Fi}$ ) by enabling the cost based offloading mechanism. Figure 5 illustrates the offloading delay for each attempt.

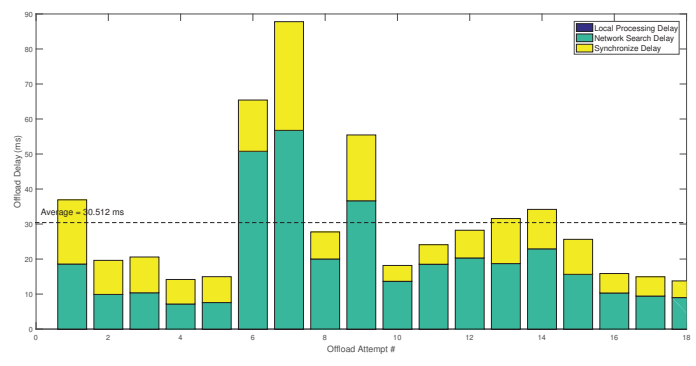

Fig. 5: User Offload Delay

The experiment results verify that average offloading delay is $30.5 \mathrm{~ms}$. Here, the offloading delay is mainly caused by network searching delay (i.e. time to identify compatible networks) and synchronization delay (i.e. time to exchange the user informations). Both of these delays are depending on the performance of the communication channel between controllers. The local processing delay at the controllers is almost negligible for all the attempts.

\section{CONCLUSION}

In this paper, we have proposed an SDN-based operatorassisted multicontroller offloading platform for $5 \mathrm{G}$ mobile networks. Our proposal extended the single-controller singleoperator offload schemes to multi-controller multi-operator networks. In addition, the proposed platform is independent of data offloading mechanism and the operator has the flexibility to select the offload mechanism. A proof-of-concept prototype is implemented by using Floodlight SDN controller and the RESTful architecture. We designed a test network on Mininet platform and analyzed for performance and feasibility. The experiment results verified that the proposed platform was functional in multi-controller networks. Moreover, the platform supported the interoperability of different offloading mechanisms.

\section{ACKNOWLEDGMENT}

This work has been performed in the framework of the SECUREConnect (Secure Connectivity of Future Cyber-Physical Systems) and Towards Digital Paradise projects. This research is funded by Academy of Finland and TEKES, Finland.

\section{REFERENCES}

[1] G. Iosifidis, L. Gao, J. Huang, and L. Tassiulas, "An Iterative Double Auction for Mobile Data Offloading," in Modeling \& Optimization in Mobile, Ad Hoc \& Wireless Networks (WiOpt), 2013 11th International Symposium on. IEEE, 2013, pp. 154-161.

[2] M. Liyanage, A. Gurtov, and M. Ylianttila, Software Defined Mobile Networks (SDMN): Beyond LTE Network Architecture. John Wiley \& Sons, 2015.

[3] A. Y. Ding, J. Crowcroft, and S. Tarkoma, "Poster: SoftOffload: A Programmable Approach Toward Collaborative Mobile Traffic Offloading," in Proceedings of the 12th annual international conference on Mobile systems, applications, and services. ACM, 2014, pp. 368-368.

[4] M. Amani, T. Mahmoodi, M. Tatipamula, and H. Aghvami, "SDN-Based Data Offloading for 5G Mobile Networks," ZTECOMMUNICATIONS, p. 34, 2014.

[5] J. Okwuibe, M. Liyanage, and M. Ylianttila, "Provider Assisted Wi-Fi Offloading Leveraging on SDN," in European Wireless 2016 (EW2016), Oulu, Finland, May 2016, pp. 43-48.

[6] J. Cho, B. Nguyen, A. Banerjee, R. Ricci, J. Van der Merwe, and K. Webb, "SMORE: Software-Defined Networking Mobile Offloading Architecture," in Proceedings of the 4th workshop on All things cellular: operations, applications, \& challenges. ACM, 2014, pp. 21-26.

[7] F. Rebecchi, M. D. De Amorim, V. Conan, A. Passarella, R. Bruno, and M. Conti, "Data Offloading Techniques in Cellular Networks: A Survey," IEEE Communications Surveys \& Tutorials, vol. 17, no. 2, pp. 580-603, 2015.

[8] M. Amani, T. Mahmoodi, M. Tatipamula, and H. Aghvami, "Programmable Policies for Data Offloading in LTE Network," in 2014 IEEE International Conference on Communications (ICC). IEEE, 2014, pp. 3154-3159. 